\title{
Vomiting, CTCAE
}

National Cancer Institute

\section{Source}

National Cancer Institute. Vomiting, CT CAE. NCI Thesaurus. Code C57896.

A disorder characterized by the reflexive act of ejecting the contents of the stomach

through the mouth. 